\title{
THEORY AND PRACTICE FOR MODELLING THE BROADBAND ACQUISITION IN HF TRANSMISSIONS
}

\author{
B. Pérez-Díaz ${ }^{\dagger}$, S. Zazo*, I. Pérez-Álvarez ${ }^{\dagger}$, J. López-Pérez ${ }^{\dagger}$, \\ L. Melián Gutiérrez ${ }^{\dagger}$ E. Jiménez-Yguácel ${ }^{\dagger}$ \\ $\uparrow$ Instituto para el Desarrollo Tecnológico y la Innovación en Comunicaciones (IDeTIC). Universidad de Las Palmas de G.C. \\ Campus de Tafira, 35017-Las Palmas G.C. (Spain).e-mail: bperez@idetic.eu \\ * Universidad Politécnica de Madrid (UPM). Dpto. SSR, E.T.S. Ingenieros de Telecomunicación. Ciudad Universitaria, \\ 28040-Madrid (Spain). e-mail: santiago@gaps.ssr.upm.es
}

Keywords: HF Modem, Wideband Acquisition, Interference Mitigation Estimation, Analog to Digital Converter, Signal to Interference Noise Ratio.

\begin{abstract}
Traditional use of the HF band is limited mostly to $3 \mathrm{KHz}$ for both voice and data transmission, and therefore, most of the transceivers bandwidths are also adapted to this profile. However, there are some initiatives that clearly state that much broader bandwidths may be use with the corresponding data rate increase and performance improvement in the HF band $[1,5]$. Due to the increased bandwidth, a broadband transceiver receiver has to cope with a broader acquisition bandwidth with strong signals that degrade the Analog to Digital Converter (ADC) performance while receiving our (often) much weaker desired signals. This current paper has three main steps in order to evaluate under which circumstances the degradation is noticeable regarding the interchannel separation and the powers ratio. Indeed, our objective is to determine how much the interference has to be mitigated in the analog domain in order to split its cancellation partly before the ADC, and partly after it in the digital domain where much powerful techniques are available.
\end{abstract}

\section{Introduction}

Our group, IDeTIC at the Universidad de Las Palmas de Gran Canaria (ULPGC) and GAPS at the Universidad Politécnica de Madrid (UPM), has been working on multicarrier HF transmissions for several years leading to the development of a proprietary system denoted as HFDVL (HF Data + Voice Links) that permits interactive digital voice transmission along with data communications reaching data rates up to $8640 \mathrm{bps}$ in the $3 \mathrm{kHz}$ bandwidth, as described in several publications (for instance [4]).

Mostly, HF band transmissions bandwidth are restricted to $3 \mathrm{kHz}$ for both voice and data transmissions, and therefore, that is the typical commercial transceivers bandwidth. However, there are some initiatives that clearly state that much broader bandwidths may be use with the corresponding data rate increase and performance improvement. For instance, [1] describes some of the features of this tendency in the framework of the MIL-STD-188-141C, which is likely to include specifications for HF radio pass bands of (nominally) 6,12 , and $24 \mathrm{kHz}$ with data rates up to $64 \mathrm{kbps}$. Also, there are already commercial transceivers including the 4-ISB mode as a transition phase to this concept. On the other hand, the authors of this paper have some experience with OFDM transmissions with arbitrary bandwidths [5] where the number of carriers is variable depending on the desired data rate observing very satisfactory performance. Although single carrier modulations are not so flexible, modern powerful equalization and coding schemes make these wider band transmissions also feasible and reliable. Our experience in real links is based on our own transceiver capable of transmitting simultaneously a set of up to 16 narrow band signals within $1 \mathrm{MHz}$ bandwidth [3]. From the very beginning, we noticed that expected performance was degraded by the presence of strong interferers located in channels even quite far of our assigned channel but still within the acquisition band. This degradation comes from the fact that the interference dominates the aggregate signal after the Automatic Gain Control (AGC) to accommodate the dynamic range to the $\mathrm{ADC}$, and therefore, there is a reduction on the effective number of bits used to represent the desired signal. We observed that the quantization noise of the interferer spreads all over the band and if the power of interference is strong enough, it exceeds the thermal noise and even the desired signal itself. Two accompanying papers are dealing with different strategies in the analog domain to mitigate this effect in case where just one antenna is available or using beamforming principles and therefore set the ADC ( $b$ bits) to work under standard conditions [6,7]. This paper addresses the evaluation under which circumstances the degradation is noticeable taking into account the interchannel separation and the powers ratio. Our aim is to determine how much interference has to be mitigated before the ADC (analog domain) to make a conversion without excessive artifacts generated by interferences.

The paper can be divided into three main parts. First of all, the developed theoretical analysis will be presented approximating both signals (desired and interference) as sinusoids with different amplitudes embedded in thermal noise. In a second part, a flexible simulator to study this problem under all the perspectives will be shown, and finally, the experiment that was developed to corroborate these results using real hardware will be described. 


\section{Mathematical Analysis}

The general approach is described in the following figure showing schematically the base band spectra of involved signals in the range $[0, B] \mathrm{Hz}$ :

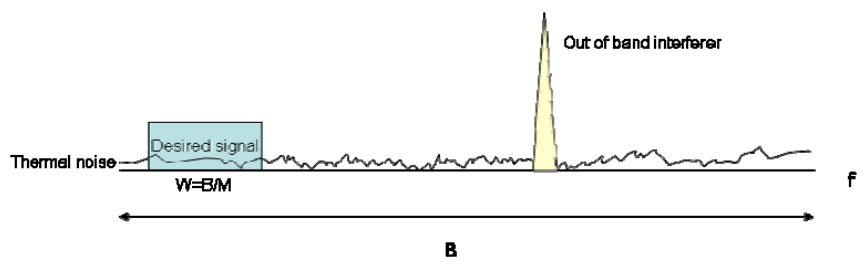

Figure 1: Simplified schematic view in broadband acquisition

$B$ : bandwidth acquisition. We suppose that the original bandpass signal is shifted to generate a complex base band version.

$W=B / M$ : desired signal bandwidth. We suppose an integer ratio $(M)$ between the bandwidth acquisition and $W$ to simplify the decimation process. In HF, where typically $\mathrm{W}=3 \mathrm{kHz}, M$ will represent the number of standard channels within the captured bandwidth.

$x(t)$ is the desired bandlimited signal characterized by a certain power $\left(\sigma_{\mathrm{x}}^{2}\right)$ and a certain Peak to Average Ratio (PAR) denoted as $P A R_{x}$ and defined as:

$$
\operatorname{PAR}_{x}=\frac{\max \left(\|\left. x(t)\right|^{2}\right)}{\sigma_{x}^{2}}
$$

$n(t)$ is the thermal noise characterized as an additive white (within the acquisition band) Gaussian noise with flat spectrum density $N_{0}$. The power becomes $\sigma_{\mathrm{n}}{ }^{2}=B \cdot N_{0}$

$i(t)$ is the interference bandlimited signal characterized by a certain power $\left(\sigma_{\mathrm{i}}^{2}\right)$ and a certain Peak to Average Ratio (PAR) denoted as $P A R_{i}$.

$r(t)=x(t)+i(t)+n(t)$ is the aggregated signal.

The SNR measured within the signal bandwidth $W$ is defined

$$
S N R=\frac{\sigma_{x}^{2}}{W N_{0}}=\frac{\sigma_{x}^{2}}{\frac{W}{B} \sigma_{n}^{2}}
$$

Once we have the base band signal, the part of the receiver where we are currently interested can be represented schematically in the following figure:

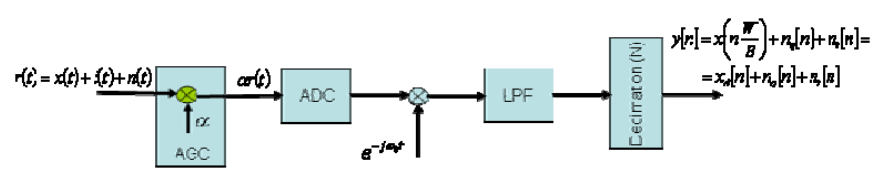

Figure 2: Standard broadband receiver (acquisition part)

For this study:

AGC: Automatic Gain Control. In real applications this is typically an adaptive filter to maintain the time varying acquired signal within the dynamic range of the ADC denoted as $[-G,+G]$. In this case we will examine the full register of $r(t)$ and multiply the incoming signal by a factor $\alpha$ that guarantees the saturation with a fixed probability (for instance $1 \%$ ). The behaviour is defined as follows:

$$
\text { Find } \alpha \mid P\{|\alpha r(t)|>G\}=0.01
$$

The ADC is represented by a uniform law with $b$ bits quantifying the input signal within the range $[-G,+G]$. Therefore, we will have $2^{b}$ steps while the step size is $\Delta$ :

$$
\Delta=\frac{2 G}{2^{b}-1} \approx \frac{2 G}{2^{b}}
$$

Now, in the digital domain, we shift the desired signal until we get a low pass representation of the desired signal (centred around 0 ). We filter out the rest of the signals using a digital low pass filter with bandwidth $\pm W / 2$ and we decimate by a factor of $M$ in order to represent the desired signal at Nyquist rate.

The output of this block diagram (Figure 2) denoted as $y[n]$ has three components:

- $\quad x_{d}[n]=x(n B / W)$ denoted as the desired signal at Nyquist rate and with amplitudes as real numbers (no quantification).

- $\quad n_{q}[n]$ as the quantization noise which can be considered for practical purposes as a white noise uncorrelated with the signal $x_{\mathrm{d}}[n][2]^{1}$ within the desired signal bandwidth and with uniform distribution within the range $\pm \Delta / 2$. This noise contribution has total power $\Delta^{2} / 12$, and after decimation process the power is scaled according to the different bandwidth: $\sigma_{q}^{2}=\left(\Delta^{2} W\right) /(12 B)$.

- $\quad n_{t}[n]$ as the thermal white noise within the desired signal bandwidth respecting the Gaussian distribution after the discretization and decimation process with the power scaled according to the different bandwidth $\sigma_{t}^{2}=\left(W \sigma_{n}^{2}\right) / B$.

\subsection{Theoretical considerations}

Assuming that we have initially a vector $\mathbf{r}=\mathbf{x}+\mathbf{i}+\mathbf{n}$ with $L$ samples representing the analog signal by a high enough (with respect to $B$ ) oversampling factor. Denoting: $m_{x}=\max (|\mathbf{x}|) \quad m_{i}=\max (|\mathbf{i}|)$ and $\eta=\mathrm{s}(\mathbf{n})$ where operator $\mathrm{s}(\cdot)$ represents the 0.99 quantile of the argument, that is element of $\mathbf{n}$ whose sorted real (or imaginary) part corresponds with the $0.99 \mathrm{~L}$ entry (notice that 0.99 represents the $1-0.01$ saturation probability defined just as an example). Theoretically we have:

$$
0.01=Q\left(\frac{\eta}{\sqrt{\sigma_{n}^{2}}}\right) \rightarrow \eta=\sqrt{\sigma_{n}^{2}} Q^{-1}(0.01)
$$

Parameter $\alpha$ can be approximated as follows

$$
\begin{aligned}
& \alpha\left(m_{x}+m_{i}+\eta\right)=G \rightarrow \alpha=\frac{G}{m_{x}+m_{i}+\eta}= \\
& =\frac{G}{\sqrt{\sigma_{x}^{2} P A R_{x}}+\sqrt{\sigma_{i}^{2} P A R_{i}}+\eta}
\end{aligned}
$$

Finally, the Signal to Noise and Interference ratio (SINR) of the discretized and decimated $r[n]$ signal now denoted as $y[n]$

\footnotetext{
${ }^{1}$ This model is asymptotically valid for small step size with respect to the signal dynamic range (we have to pay attention to this in our simulations).
} 


$$
\begin{aligned}
& \operatorname{SINR}=\frac{\alpha^{2} \sigma_{x}^{2}}{\alpha^{2} \sigma_{t}^{2}+\sigma_{q}^{2}}=\frac{\sigma_{x}^{2}}{\sigma_{t}^{2}+\frac{\sigma_{q}^{2}}{\alpha^{2}}}= \\
& =\frac{\sigma_{x}^{2}}{\sigma_{t}^{2}+\frac{\left(\sqrt{\sigma_{x}^{2} P A R_{x}}+\sqrt{\sigma_{i}^{2} P A R_{i}}+\eta\right)^{2} \sigma_{q}^{2}}{G^{2}}}
\end{aligned}
$$

Obviously, if $\sigma_{t}^{2}>\frac{\left(\sqrt{\sigma_{x}^{2} P A R_{x}}+\sqrt{\sigma_{i}^{2} P A R_{i}}+\eta\right)^{2} \sigma_{q}^{2}}{G^{2}}$ the quantization noise is negligible with respect to the interference signal and therefore the interferer affects very little the performance. However, when $\sigma_{t}^{2} \approx \frac{\left(\sqrt{\sigma_{x}^{2} P A R_{x}}+\sqrt{\sigma_{i}^{2} P A R_{i}}+\eta\right)^{2} \sigma_{q}^{2}}{G^{2}}$ the degradation can be very noticeable. Introducing the step size value we get, $\sigma_{t}^{2} \approx \frac{1}{3} \frac{\left(\sqrt{\sigma_{x}^{2} P A R_{x}}+\sqrt{\sigma_{i}^{2} P A R_{i}}+\eta\right)^{2}}{2^{2 b} B / W}=\frac{1}{3} \frac{\left(\sqrt{\sigma_{x}^{2} P A R_{x}}+\sqrt{\sigma_{i}^{2} P A R_{i}}+\eta\right)^{2}}{M_{b w}}$, where $M_{b w}$ represent the equivalent number of quantization steps for the bandwidth $W$ in the full $B$ band. If we further assume that the interference is the dominant term in the numerator we have $\sigma_{t}^{2} \approx \frac{\sigma_{i}^{2} P A R_{i}}{3 M_{b w}}$. Considering this equation for the particular case where the interference is a sinusoid, we get $\sigma_{t}^{2} \approx \frac{1}{3} \frac{\sigma_{i}^{2} P A R_{i}}{M_{b w}}=\frac{2}{3} \frac{\sigma_{i}^{2}}{M_{b w}}$. In that case, the condition for noticeable effect becomes (where INR stands for Interference to Noise ratio):

$$
\begin{aligned}
& \operatorname{INR}(d B)=10 \log _{10}\left(\frac{\sigma_{i}^{2}}{\sigma_{t}^{2}}\right) \geq 10 \log _{10}\left(\frac{3}{2} M_{b w}\right) \approx \\
& \approx 1.76+6.02 b+10 \log _{10}(B / W)
\end{aligned}
$$

It can be noticed from this equation that an interferer located close to the receiver transmitting kilowatts may be very harmful to the receiver whose sensitivity is around microwatts, even for very expensive ADCs with 16 bits. In case there were several interferers, the limit applies to the aggregate power. The equation also states that for every extra bit we have, we get an extra $6 \mathrm{~dB}$ margin as protection against the interferences.

In the sequel we are going to reach an approximate of the SINR in terms of the SNR (which includes the thermal and quantification noise). In order to make the analysis tractable we will assume that both the desired and interference signal are pure sinusoids (amplitude $A$ and $I$ respectively)

$$
\begin{aligned}
& S N R=\frac{\alpha^{2} \sigma_{x}^{2}}{\alpha^{2} \sigma_{t}^{2}+\sigma_{q}^{2}}=\frac{\sigma_{x}^{2}}{\sigma_{t}^{2}+\frac{\sigma_{q}^{2}}{\alpha^{2}}}=\frac{\sigma_{x}^{2}}{\sigma_{t}^{2}+\frac{\left(\sqrt{\sigma_{x}^{2} P A R_{x}}+\eta\right)^{2} \sigma_{q}^{2}}{G^{2}}}= \\
& =\frac{A^{2} / 2}{\sigma_{t}^{2}+\frac{1}{3} \frac{\left(\sqrt{\sigma_{x}^{2} P A R_{x}}+\eta\right)^{2}}{M_{b w}}}
\end{aligned}
$$

if we assume that the noise contribution is not too high

$$
S N R=\frac{A^{2} / 2}{\sigma_{t}^{2}+\frac{1}{3} \frac{\left(\sqrt{\sigma_{x}^{2} P A R_{x}}+\eta\right)^{2}}{M_{b w}}}=\frac{A^{2}}{2 \sigma_{t}^{2}+\frac{2}{3} \frac{A^{2}}{M_{b w}}}
$$

Let us express previous equation as the inverse of SNR

$$
S N R^{-1}=\frac{2 \sigma_{t}^{2}}{A^{2}}+\frac{2}{3 M_{b w}}
$$

On the other hand, the full expression of SINR

$$
\operatorname{SINR}=\frac{\sigma_{x}^{2}}{\sigma_{t}^{2}+\frac{\left(\sqrt{\sigma_{x}^{2} P A R_{x}}+\sqrt{\sigma_{i}^{2} P A R_{i}}+\eta\right)^{2}}{3 M_{b w}}}
$$

can be further simplified if we assume that the interference power is much larger than the desired signal and noise:

$$
\operatorname{SINR}=\frac{A^{2} / 2}{\sigma_{t}^{2}+\frac{I^{2}}{3 M_{b w}}}
$$

Again, in terms of the inverse we have

$$
\operatorname{SINR}^{-1}=\frac{2 \sigma_{t}^{2}}{A^{2}}+\frac{2 I^{2}}{3 A^{2} M_{b w}}+\frac{2}{3 M_{b w}}-\frac{2}{3 M_{b w}}
$$

where the last two terms are added and subtracted in order to figure out a relationship with SNR

$$
\begin{aligned}
& S I N R^{-1}=S N R^{-1}+\frac{2 I^{2}}{3 A^{2} M_{b w}}-\frac{2 A^{2}}{3 A^{2} M_{b w}}= \\
& =S N R^{-1}+\frac{2\left(I^{2}-A^{2}\right)}{3 A^{2} M_{b w}} \approx S N R^{-1}+\frac{2 I^{2}}{3 A^{2} M_{b w}}
\end{aligned}
$$

After a straightforward manipulation we have:

$$
\frac{1}{S I N R}=\frac{1}{S N R}+\frac{2 I^{2}}{3 A^{2} M_{b w}}=\frac{3 A^{2} M_{b w}+2 I^{2} S N R}{3 A^{2} M_{b w} S N R}
$$

In logarithmic units we get:

$$
\begin{aligned}
& \operatorname{SINR}(d B)=S N R(d B)+10 \log _{10} \frac{3 A^{2} M_{b w}}{3 A^{2} M_{b w}+2 I^{2} 10^{\frac{S N R(d B)}{10}}}= \\
& =S N R(d B)-10 \log _{10}\left(1+\frac{2 I^{2} 10^{\frac{S N R(d B)}{10}}}{3 A^{2} M_{b w}}\right)
\end{aligned}
$$

Let us plot this equation in Figure 3 for a realistic $\mathrm{SNR}=10 \mathrm{~dB}$ as a function of the number of bits $(b)$ for different values of interference power desired signal ratio:

$$
\frac{\sigma_{i}^{2}}{\sigma_{x}^{2}}=\frac{I^{2}}{A^{2}}
$$

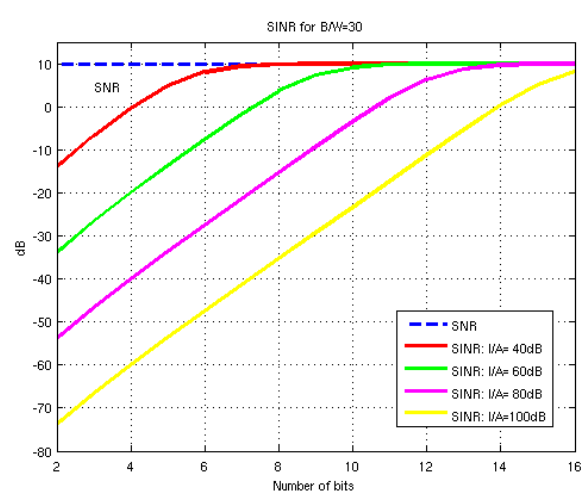

Figure 3: SINR versus number of bits

We can conclude that even for very large number of bits (14 or 16) the degradation is very important for high $I / A$ ratio and makes the system unfeasible. It is worthy to observe the last term in equation (16): 


$$
10 \log _{10}\left(1+\frac{2 I^{2} 10^{\frac{S N R(d B)}{10}}}{3 A^{2} M_{b w}}\right)
$$

If the denominator is high enough with respect to the numerator, $\operatorname{SINR}(d B) \rightarrow S N R(d B)$ but in the opposite case (the most interesting regarding this study) we reach a very conclusive result.

$$
\begin{gathered}
\operatorname{SINR}(d B)=S N R(d B)-10 \log _{10}\left(1+\frac{2 I^{2} 10^{\frac{S N R(d B)}{10}}}{3 A^{2} M_{b w}}\right) \approx \\
\approx S N R(d B)-10 \log _{10}\left(\frac{2 I^{2} 10^{\frac{S N R(d B)}{10}}}{3 A^{2} M_{b w}}\right)= \\
=S N R(d B)-S N R(d B)-10 \log _{10}\left(\frac{2}{3}\right)-10 \log _{10}\left(\frac{\sigma_{i}^{2}}{\sigma_{x}^{2}}\right)+10 \log _{10}\left(M_{b w}\right) \\
=1.76+6.02 b+10 \log _{10}(B / W)-10 \log _{10}\left(\frac{\sigma_{i}^{2}}{\sigma_{x}^{2}}\right)= \\
=1.76+6.02 b+10 \log _{10}(B / W)+\sigma_{x}^{2}(d B)-\sigma_{i}^{2}(d B)
\end{gathered}
$$

Corroborating the previous result, we again conclude that for a certain desired signal we gain $6 \mathrm{~dB}$ for every extra bit we have but we loose as much as the power of the interference in $\mathbf{~} \mathrm{B}$.

\section{Model verification}

We have made some simulations obtaining quite accurate results, although we have noticed that for most of the interesting cases, when the interference is quite large, the signal amplitude is reduced so much that the quantization noise is strongly correlated with the signal itself neither it is uniformly distributed. We can show the theoretic and simulated results for $\mathrm{SNR}=10 \mathrm{~dB}$ and $I / A=60 \mathrm{~dB}$.

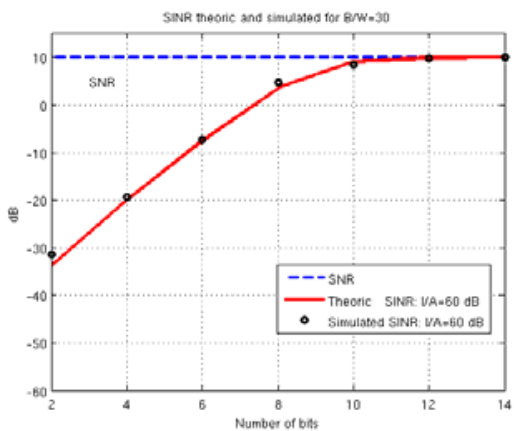

Figure 4: SINR for $\mathrm{I} / \mathrm{A}=60 \mathrm{~dB}$ versus num. of bits

It can be observed a good match for both low and high number of bits. It is clear that for high number of bits, the model of the quantization noise is valid while for very low number of bits the system behaves as:

$$
S I N R \rightarrow 10 \log _{10}\left(\frac{\alpha^{2} \sigma_{x}^{2}}{\left(\Delta^{2} / 12\right)(W / B)}\right)
$$

This equation is equivalent to the previous one but present more clearly what is happening: signal power is reduced by the AGC factor while the dominant noise is the quantization noise coming from the interference signal. There are also other contributions from the quantization process of the desired signal but the term should be avoided because, in fact, the distortion and the error are strongly correlated with the signal. Fortunately, this term decreases as the signal does due to the AGC factor and previous equation is an accurate approximation.

\section{Hardware experiment}

An experiment was built to corroborate these results using real hardware. The ADC chosen was the AKM 5357VT chip that incorporates the Edirol UA-1EX soundcard. This circuit is a 24 bits stereo $\Sigma \Delta \mathrm{ADC}$ with a $96 \mathrm{kHz}$ max sampling rate. We chose to study a soundcard ADC because it facilitates to (19) capture audio signals and process them directly with MATLAB software suite.

Initially, a setup, as depicted in Figure 5, was built to relate the converted signal (digital format) to the analog one in MATLAB and also to establish ADC full scale (FSD). As you can see, a function generator outputs a sinusoid signal that pass through a variable attenuator (10dB step) before entering soundcard LINE IN. An oscilloscope in parallel is used to check actual ADC input signal level.

A signal was recorded for every step attenuator size at $9600 \mathrm{~Hz}$ sampling rate and processed in MATLAB. A sinusoid frequency of $953 \mathrm{~Hz}$ was chosen as the desired signal in order to avoid correlation between the quantization noise and the signal regarding the $9600 \mathrm{~Hz}$ sampling rate. At the end of the experiment, it was reckoned a $+3 \mathrm{dBm}(890 \mathrm{mVpp})$ ADC full scale and also it was checked that SNR is proportional to step attenuation with a FSD SNR of $78 \mathrm{~dB}$ $(-75 \mathrm{dBm}$ total noise power). In order to simplify the experiment only 15 bits were read from the soundcard, as well as, LINE IN soundcard input impedance was forced to $50 \mathrm{ohms}$ to make easier signal generator power output reading and fix variable attenuator impedance terminations.

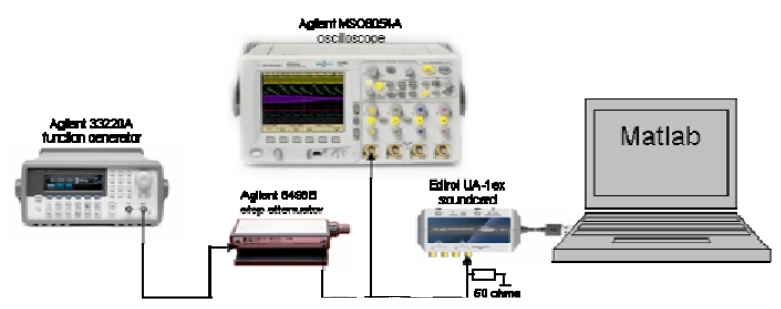

Figure 5: First setup to evaluate ADC

A second setup was built to be able to insert an interference to corroborate equation (19) SINR relationship. As you can see in Figure 6, another function generator behaves as an interferer and their outputs are combined before entering soundcard LINE IN. There is also another function generator that acts as a white noise source. An interference frequency of $1113 \mathrm{~Hz}$ was chosen to avoid correlation also due to the sampling rate. In this experiment, the interference was fixed at $3 \mathrm{~dB}$ below FSD $(0 \mathrm{dBm})$ to avoid $\mathrm{ADC}$ saturation and the desired signal was stepped down from $0 \mathrm{~dB}$ to $100 \mathrm{~dB}$ interference to signal ratio to facilitate the procedure. 


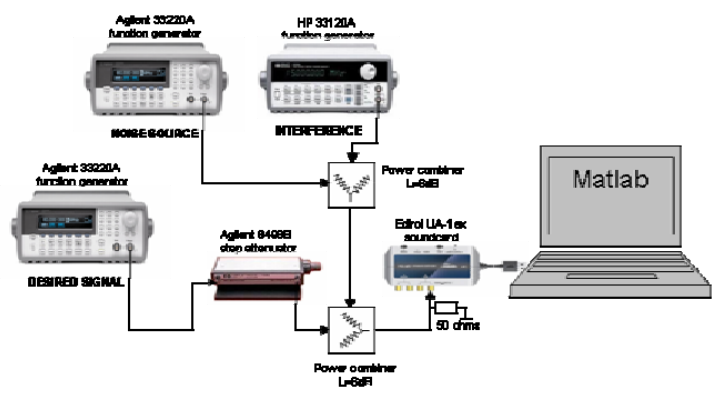

Figure 6: Second setup to evaluate SINR

Figure 7 depicts SINR measured in the signal bandwidth for a $-40 \mathrm{~dB}$ signal to interference ratio regarding number of ADC bits. It clearly shows a good match between theoretic and measured curves and it also corroborates that for every extra bit there is a $6 \mathrm{~dB}$ protection margin against interferences. It also shows that below 8 bits quantization noise is the main noise source and above that dominates the thermal noise.

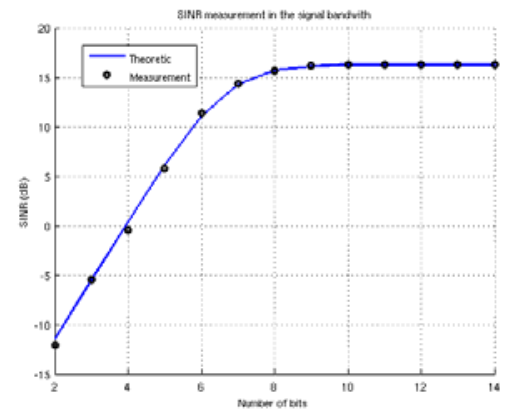

Figure 7: SINR measurement versus number of bits

Figure 8 and Figure 9 depicts desired and interferer signal spectra for 4 bits and 8 bits ADC respectively. It can be noticed that the quantization noise is higher for 4 bits where less bits are dedicated to the quantization process.

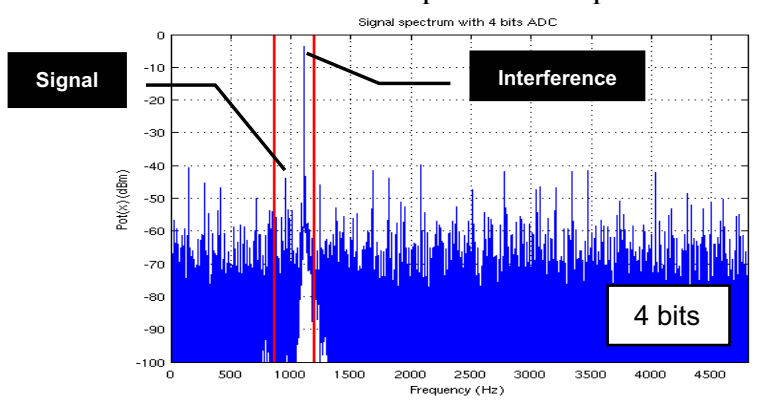

Figure 8: Signal spectrum with 4 bits ADC

\section{Conclusions}

In this paper we have emphasized that broadband acquisitions must foresee strong signals inside the acquisition bandwidth that degrades ADC conversion of desired signal. A mathematical analysis of an ADC transceiver receiver with jointly desired and interference signal was developed to conclude with equation (19). It states that for a certain desired signal we gain $6 \mathrm{~dB}$ for every extra bit we have but we loose as much as the power of the interference in $\mathrm{dB}$. Subsequently a simulator to verify the model was presented and a real experiment was built that corroborates the theoretical analysis. Some interesting alternatives to deal with interferences in multiband HF broadband transmissions are also presented to the meeting by our group, as [6] that approaches it using analog beamforming principles, and [7] that combines an analog notch filter with Higher Order Crossing statistics in the digital domain.

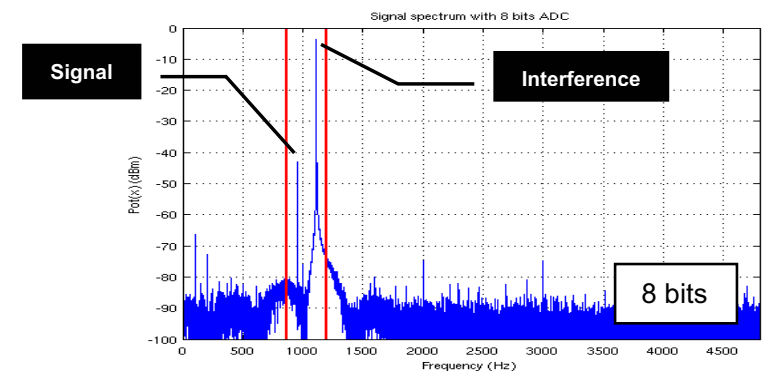

Figure 9: Signal spectrum with 8 bits ADC

\section{Acknowledgements}

This work was supported by the Spanish Ministry of Science and Innovation under grants TEC2010-21217-C02 CR4HFDVL, and CONSOLIDER-INGENIO 2010 CSD2008-00010 COMONSENS.

\section{References}

[1] E. E. Johnson. "Performance Envelope of Broadband HF Data Waveforms", (WBHF Applications), Proceedings of MILCOM 2009, IEEE*, Boston, MA, Oct 2009.

[2] Marco, D.; Neuhoff, D. L. "The Validity of the Additive Noise Model for Uniform Scalar Quantizers", IEEE Transactions on Information Theory, Vol. IT-51, No. 5, pp. 1739-1755, May 2005.

[3] Pérez-Díaz, B.; Jiménez-Yguacel, E.; López-Pérez, J.; Pérez-Álvarez, I.; Zazo, S.; Mendieta, E. "Design and construction of a broadband $(1 \mathrm{MHz})$ digital HF transceiver for multicarrier and multichannel modulations", IRST 2009. Edinburgh, U.K., April 2009.

[4] Santana-Sosa H. et al. "Performance over a Real Link of a HF Software Radio Modem for Interactive Digital Voice Communications", IRST 2006. London, UK.

[5] Zazo-Bello, S.; Raos, I.; López-Pérez, J.; Pérez-Álvarez, I.; Pérez-Díaz, B.; Jiménez-Yguacel, E. "Asynchronous and Variable Data Rate OFDM Modem for RADAR Data Transmission". IRST 2009. Edinburgh, U.K.; pp. 220-224, April 2009.

[6] Zazo-Bello, S.; Pérez-Álvarez, I.; López-Pérez, J.; PérezDíaz, B.; Jiménez-Yguacel, E.; Melián-Gutiérrez L.; Sanz-González, J.L. "Spatial Domain Mitigation of Out of Band Strong Interferences in HF Wideband Acquisition Using Analog Beamforming Principles". Submitted to IRST 2012. York, U.K., May 2012.

[7] Zazo-Bello, S.; Sanz-González, J.L.; Pérez-Díaz, B.; Pérez-Álvarez, I.; López-Pérez, J.; Melián-Gutiérrez L. "Analog Mitigation of Out of Band Strong Interferers in Wide Band Acquisition for Multiband HF Transmissions". Submitted to IRST 2012. York, U.K., May 2012. 